\title{
Mixed-form extremum problem statements for small-deformation elastostatics
}

\author{
J.E. Taylor
}

\begin{abstract}
In situations outside those identified with routine elastic structural analysis, there is often a need for formulation in mixed form. Small-deformation elastostatics, expressed in terms of stress, strain, and displacement, is described here in the form of either of two complementary constrained-extremum problems. The set of governing equations and boundary conditions of elastostatics are obtained by an interpretation of the generalized "necessary conditions" for each of these fully mixed variational formulations. While the objectives in the problem statements are bilinear and therefore nonconvex, a simple proof is available to confirm that the solution to these conditions is an extremizer. Extensions of the basic formulation, obtained by the introduction of constraints or optimal relaxations, simulate constitutively nonlinear systems. The mixed formulations also provide a convenient representation of the mechanics requirements in connection with structural optimization.
\end{abstract}

Key words continuum structures, mixed-form models, variational formulations, elastostatics, optimal design

1

\section{Introduction}

Within the problem setting governed by elastostatics, any performance criterion to be accounted for in optimal design must be represented, necessarily, in terms of one or a combination of stress, strain, and displacement. Therefore in order to be broadly applicable for design, all three measures should appear explicitly in a variational formulation for the structural analysis. One might expect this

Received: 14 March 2002

Published online: 30 June 2003

(C) Springer-Verlag 2003

J.E. Taylor

Department of Aerospace Engineering, University of Michigan - Ann Arbor, MI 48109-2118, USA

e-mail: janos@umich.edu breadth of capability to be especially useful in the case of multicriterion design. Besides concern for design, in certain situations the use of such a mixed form may be indicated as the basis for computational determination of structural response alone. A need for precision in the prediction of stresses, beyond what can be expected in the typical displacement-based model, is a case in point. The usefulness of the fully mixed-form characterization may be realized either analytically in connection with the development of a new model or in implementations for numerical treatment.

Roberts and Thomas (1991) defined a mixed model as any formulation in which two or more field variables are present. Commonly the functional stated to represent a mixed model is described as the sum of the potential energy or complementary potential energy plus terms identified as "multipliers times constraints". Such functionals are termed Lagrangian, and the implication is that the model is one or another form of extension of the classical min principles. By contrast, the objective in the mixed formulations of this paper is simply the integral of the product of two symmetric two-tensors (to be identified ex post facto as stress and strain). Strain energy or complementary strain energy appear there within the set of constraints.

In the next section, two unique mixed-form variational formulations for kinematically linear elastostatics of continuum structures are stated, each in the form of a constrained-extremum problem. The emphasis is on problem formulations. Implementation for computational solutions is not addressed. Roberts and Thomas (1991) have presented a thorough treatment of finite-element methods for mixed models. These problem statements have the mechanics expressed in fully mixed form, i.e. in terms of stress, strain, and displacement, in the two complementary formulations. In both cases, the objective functional is bilinear, the integral of stress times strain mentioned above. Constitutive relations are implicit in the models, in the form of a required stress or strain potential. Governing equations for the elastostatic boundary-value problems are recognized through an interpretation of the necessary conditions (generalized Kurash-Kuhn-Tucker conditions) for these models. An argument to establish sufficiency of these conditions is set 
forth in the Appendix. The relationship between the familiar Reissner and $\mathrm{Hu}-$ Washizu 'mixed principles' and these models is discussed. All developments are described for continuum structures, but of course the formulations apply as well for discrete structural systems.

The basic complementary extremum problem statements provide a ready platform for extension to broader theory, i.e. to obtain models that account for additional aspects of mechanics or design. A second objective of this paper is to indicate how this quality may be exploited to advantage. Formulations for elastostatics with weakening or locking material properties are described as examples; these effects are introduced through the addition of local constraints. The problem of minimum compliance design within bounds on stress is presented to show how the mixed-formulation may be incorporated into the design setting. Predictions of the optimal material stiffening and material relaxation designs for a structure composed of stress-limited or strain-limited material are treated as examples of another category of design problem.

\section{2}

\section{The basic problem statements}

The models termed "basic" and described in this section correspond in effect to standard small-strain elasticity for static systems. Several elaborations on these models are presented below, under the heading Applications in analysis and design. As a first step in this description of a fully general mixed formulation for elastostatics, consider the following symbolic statement for a constrained max problem:

$\max _{\sigma, \varepsilon, u} \int_{\Omega} \sigma_{i j} \varepsilon_{i j} \mathrm{~d} V$

subject to

[PI ] $\left[\int_{\Omega} f_{k} u_{k} \mathrm{~d} V+\int_{\Gamma_{t}} t_{k} u_{k} \mathrm{~d} S\right]-\bar{W} \leq 0$,

$\int_{\Omega} U_{0 \sigma}(\sigma) \mathrm{d} V-\bar{U}_{\sigma} \leq 0$,

$\varepsilon_{i j}-\frac{1}{2}\left(u_{i, j}+u_{j, i}\right)=0$.

Note that no reference is made to an explicit requirement of equilibrium (or virtual work equation), or to constitutive relations. $\sigma_{i j}$ and $\varepsilon_{i j}$ represent as yet undesignated symmetric, real valued two-tensors, and $u_{k}$ a real valued vector, etc. Data for [PI] are loads $f_{k}$ and $t_{k}$, bound values $\bar{W}$ and $\bar{U}_{\sigma}$, and function $\bar{U}_{0 \sigma}(\sigma)$, the latter meeting the usual requirements for a stress potential. (For the sake of brevity in this development, where displacements are prescribed they are taken to have the value zero). Admissible $\sigma_{i j}, \varepsilon_{i j}$, and $u_{k}$ are required to be continuous and to have unique derivatives, i.e. consistent with the statements below of necessary conditions. These conditions correspond to the generalized Kurash-KuhnTucker (KKT) conditions for problem [PI]. Listed in the order associated respectively with variation of $\sigma, \varepsilon$, and $u$, they are $\left(\Lambda_{f}, \Lambda_{\sigma}\right.$, and $\lambda_{i j}$ are multipliers associated in the order listed with the three constraints of $[\mathrm{PI}])$ :

$\left.\begin{array}{l}\varepsilon_{i j}+\Lambda \frac{\partial U_{o \sigma}}{\partial \sigma_{i j}}=0, \\ -\sigma_{i j}+\lambda_{i j}=0, \\ \Lambda_{f} f_{k}+\lambda_{i k, j}=0,\end{array}\right\} \quad x$ in $\Omega$,

and the complementarity conditions and restrictions on multipliers are

$\Lambda_{f}\left[\int_{\Omega} f_{k} u_{k} \mathrm{~d} V+\int_{\Gamma_{t}} t_{k} u_{k} \mathrm{~d} S-\bar{W}\right]=0$,

$\Lambda_{\sigma}\left[\int_{\Omega} U_{0 \sigma}(\sigma) \mathrm{d} V-\bar{U}_{\sigma}\right]=0$,

$\Lambda_{f} \geq 0, \quad \Lambda_{\sigma} \geq 0$.

A null solution to the system (5)-(11) is excluded in favor of the maximizer. Accordingly, multipliers $\Lambda_{f}$ and $\Lambda_{\sigma}$ have positive values, and from (9) and (10),

$$
\begin{aligned}
& {\left[\int_{\Omega} f_{k} u_{k} \mathrm{~d} V+\int_{\Gamma_{t}} t_{k} u_{k} \mathrm{~d} S-\bar{W}\right]=0,} \\
& {\left[\int_{\Omega} U_{0 \sigma}(\sigma) \mathrm{d} V-\bar{U}_{\sigma}\right]=0 .}
\end{aligned}
$$

Substitution of (6) into (7) and (8) provides that, up to the unknown multiplier $\Lambda_{f}$, the heretofore undesignated $\sigma$ may be recognized, through the stressequilibrium boundary-value problem statement, as a measure of stress. With $U_{\sigma}$ in the role of "stress potential", (5) comprises a statement (up to multiplier $\Lambda_{\sigma}$ ) of the constitutive relation.

To complete the interpretation of problem [PI], the multipliers are evaluated next. Suppose for this purpose that the above boundary-value problem (7) and (8) is solved for $\tilde{\sigma}_{i j}=\sigma_{i j} / \Lambda_{f}$. The equation obtained via substitution of this result into (13), i.e.

$\left[\int_{\Omega} U_{0 \sigma}\left(\Lambda_{f} \tilde{\sigma}_{i j}\right) \mathrm{d} V-\bar{U}_{\sigma}\right]=0$,

may be solved for $\Lambda_{f}$. Toward determination of the last unknown, note that after multiplication of (7) (expressed 
for the known $\tilde{\sigma}_{i j}$ ) by $u_{k}$ and integration by parts, making use of boundary condition (8), one finds

$$
\int_{\Omega} \tilde{\sigma}_{i j} \varepsilon_{i j} \mathrm{~d} V=\int_{\Omega} f_{k} u_{k} \mathrm{~d} V+\int_{\Gamma_{t}} t_{k} u_{k} \mathrm{~d} S .
$$

Also, integration of the product of (5) and $\tilde{\sigma}_{i j}$ produces

$$
\int_{\Omega} \tilde{\sigma}_{i j} \varepsilon_{i j} \mathrm{~d} V=\frac{\Lambda_{\sigma}}{\Lambda_{f}} \int_{\Omega} \tilde{\sigma}_{i j} \frac{\partial U_{0 \sigma}}{\partial \tilde{\sigma}_{i j}} \mathrm{~d} V .
$$

With $\Lambda_{f}$ and $\tilde{\sigma}_{i j}$ known, (15), (16), and (12) provide for evaluation of $\Lambda_{\sigma}$. Note that in contrast to portrayals of elastostatics in which the object is to determine response to prescribed loads, i.e. the usual form of problem statement, here the solution represents response for a specified compliance $\bar{W}$ and stress energy $\bar{U}_{\sigma}$ and load configurations $f_{k}$ and $t_{k}$. This form for the expression of elastostatics proves to be more convenient for the description of certain design problems. Finally, a proof is given in the Appendix to confirm that the solution to the above KKT conditions does in fact lead to a maximizer for the constrained max problem [PI].

A characterization of elastostatics for a variable load path is available with the introduction of the parameter $\alpha$, as indicated in the modified formulation [PI.1] below. In this case, the load path is represented via the dependence of $f_{k}(x, \alpha)$ and $t_{k}(x, \alpha)$ on $\alpha$ (linear dependence $\alpha f_{k}$ and $\alpha t_{k}$ models proportional loading, for example). The last constraint is active at the solution and so the prescribed value $\underline{\alpha}$ in fact designates a 'point on the load path'. A sequence of solutions for monotonically increasing values of $\underline{\alpha}$ may serve to predict a process or evolution of response. While it is generally not of interest in the strictly linear problem, the formulation with path-dependent loading is convenient for the treatment of nonlinear problems, e.g. in analysis in which material degradation may occur. Having the load path represented in an abstract, i.e. non-specific, form also may be useful in the treatment of optimal design.

A generalization of [PI] to accommodate the parameter $\alpha$ is stated symbolically as

$$
\max _{\sigma, \varepsilon, u, \alpha} \int_{\Omega} \sigma_{i j} \varepsilon_{i j} \mathrm{~d} V,
$$

subject to

$$
\begin{aligned}
\text { [PI.1 ] } & {\left[\int_{\Omega} f_{k}(\alpha) u_{k} \mathrm{~d} V+\int_{\Gamma_{t}} t_{k}(\alpha) u_{k} \mathrm{~d} S\right]-\underline{W} \leq 0, } \\
& \int_{\Omega} U_{0 \sigma}(\sigma) \mathrm{d} V-\bar{U}_{\sigma} \leq 0, \\
& \varepsilon_{i j}-\frac{1}{2}\left(u_{i, j}+u_{j, i}\right)=0, \\
& \underline{\alpha}-\alpha \leq 0 .
\end{aligned}
$$

Conditions governing the solution to this problem are the same as those for [PI] but for the additional requirements

$\Lambda_{f}\left[\int_{\Omega} \frac{\partial f_{k}}{\partial \alpha} u_{k} \mathrm{~d} V+\int_{\Gamma_{t}} \frac{\partial t_{k}}{\partial \alpha} u_{k} \mathrm{~d} S\right]-\Lambda_{\alpha}=0$,

$\Lambda_{\alpha}(\underline{\alpha}-\alpha)=0 \quad \Lambda_{\alpha} \geq 0$,

where $\Lambda_{\alpha}$ is the multiplier associated with the last constraint of [PI.1]. Concern in the present setting is confined to the case for which multiplier $\Lambda_{\alpha}$ has positive value and thus for which the rate measure in square brackets is positive.

We note, however, that the mechanics portrayal of formulation [PI.1] admits an interpretation about global stability, namely that where an elastostatic system may become unstable with respect to the load path, a change in sign of the quantity of (22) in square brackets from positive to negative may be identified with a loss of stability. In analytical terms, the problem does not admit a solution for which that quantity turns to negative values.

The mixed formulation is easily extended by the introduction of constraints on stress. With such constraints incorporated, the model simulates a system in which degradation of the material capacity occurs. An example is discussed in the section on applications. To elaborate first on an alternate form for the basic formulation, note that it may be convenient in some settings to deal with the elastostatics problem [ PI.1] stated in an equivalent reciprocal form (Hestenes 1966). The following reciprocal form is obtained by an appropriate interchange between objective and the load parameter constraint:

$$
\max _{\sigma, \varepsilon, u, \alpha} \alpha
$$

subject to

$$
\begin{aligned}
& \int_{\Omega} U_{0 \sigma}(\sigma) \mathrm{d} V-\bar{U}_{\sigma} \leq 0, \\
& \varepsilon_{i j}-\frac{1}{2}\left(u_{i, j}+u_{j, i}\right)=0, \\
& \underline{U}_{\sigma \varepsilon}-\int_{\Omega} \sigma_{i j} \varepsilon_{i j} \mathrm{~d} V \leq 0 .
\end{aligned}
$$$$
\text { [PI.2 ] }\left[\int_{\Omega} f_{k}(\alpha) u_{k} \mathrm{~d} V+\int_{\Gamma_{t}} t_{k}(\alpha) u_{k} \mathrm{~d} S\right]-\underline{W} \leq 0,
$$

Equivalence between the original [PI.1] and its reciprocal [PI.2] may be verified by comparison of their respective sets of governing conditions. To elaborate, suppose the data for [PI.1] is given, and that all data common to the two problem statements are the same. Then the 'equivalence' is associated with the condition that 
for a given value $\underline{\alpha}$, there exists a value of $\underline{U}_{\sigma \varepsilon}$, say $\underline{U}_{\sigma \varepsilon}^{*}$, such that the solution to [PI.2] is identical to that of [PI.1].

On another aspect of formulations, consider a comparison of the problem remaining after elimination of $\varepsilon_{i j}$ from formulation [PI], namely

$$
\max _{\sigma, u} \int_{\Omega} \sigma_{i j} u_{i, j} \mathrm{~d} V,
$$

subject to

$$
\begin{aligned}
\text { [PI.3 ] } & {\left[\int_{\Omega} f_{k} u_{k} \mathrm{~d} V+\int_{\Gamma_{t}} t_{k} u_{k} \mathrm{~d} S\right]-\bar{W} \leq 0, } \\
& \int_{\Omega} U_{0 \sigma}(\sigma) \mathrm{d} V-\bar{U}_{\sigma} \leq 0
\end{aligned}
$$

with statement of the Reissner principle (Reissner 1950). The functional commonly given to characterize the sonamed principle may be identified with the Lagrangian for problem [PI.3 ], once the multipliers have been evaluated. To summarize, the Reissner principle, commonly identified in the literature (Langhaar 1962; Washizu 1982; Shames and Dym 1985; Mura and Tatsuhito 1992; Reddy 1984; Holzapfel 1998), for example as a stationarity principle, is embodied in effect in the present setting in conjunction with a (properly posed) extremum problem. As was indicated in the introduction, having the structural mechanics expressed in extremum problem form rather than in explicit or 'stationary condition' form is generally advantageous in the treatment for optimal design. Also the extremum problem formulation for elastostatics serves to support development of finite-element or other discretization modeling for the mixed formulation of elastostatics. It is understood in regard to the latter that the "design variable" fields are to be discretized as well as the "state variable" fields.

As holds in the case of classical variational statements for elastostatics, a complementary form for a general mixed extremum problem formulation is available. Along the lines of the development above, this complementary version is described first via a statement in symbolic form for the governing constrained max problem. It is verified subsequently that the set of governing conditions for this problem again covers all requirements for statement of a small deformation model of elastostatics. The symbolic problem statement is

$$
\max _{\sigma_{i j}, \varepsilon_{i j}} \int_{\Omega} \sigma_{i j} \varepsilon_{i j} \mathrm{~d} V,
$$

subject to

$$
\begin{aligned}
{[\text { PII ] }} & \int_{\sigma_{i j}} n_{j} \bar{u}_{i} \mathrm{~d} S-\bar{W}_{\bar{u}} \leq 0, \\
& \int_{\Omega} U_{\varepsilon}\left(\varepsilon_{i j}\right) \mathrm{d} V-\bar{U}_{\varepsilon} \leq 0,
\end{aligned}
$$

$$
\begin{array}{ll}
-\sigma_{i j j}-f_{j}=0 & \text { on } \Omega, \\
-\sigma_{i j} n_{i}-t_{j}=0 & \text { on } \Gamma_{t} .
\end{array}
$$

Variables $\sigma_{i j}$ and $\varepsilon_{i j}$ are again identified simply as symmetric two-tensors, and $U_{\varepsilon}$ must qualify as a 'strain potential'. For simplicity in what follows, the union of $\Gamma_{u}$ and $\Gamma_{t}$ is supposed here to cover the boundary of structural domain $\Omega$ without overlap. Quantities and functions $\bar{W}_{\bar{u}}, \bar{U}_{\varepsilon}, f_{j}, t_{j}, \bar{u}_{i}$, and form of $U_{\varepsilon}$ are the data for problem [PII]. With $\Lambda_{\bar{u}}, \Lambda_{\varepsilon}, \gamma_{j}$, and $\beta_{j}$ to represent, in order, multipliers for the four constraints, the governing system for [PII ] is

$\sigma_{i j}-\Lambda_{\varepsilon} \frac{\partial U_{\varepsilon}}{\partial \varepsilon_{i j}}=0$,

$\varepsilon_{i j}-\frac{1}{2}\left(\gamma_{i, j}+\gamma_{j, i}\right)=0$,

and after interpretation of the boundary integrals

$\gamma_{j}=\Lambda_{\bar{u}} \bar{u}_{j} \quad$ in $\Gamma_{\bar{u}}$,

$\beta_{j}=\gamma_{j} \quad$ in $\Gamma_{t}$,

together with the conditions

$\Lambda_{\bar{u}}\left[\int_{\Gamma_{\bar{u}}} \sigma_{i j} n_{j} \bar{u}_{i} \mathrm{~d} S-\bar{W}_{\bar{u}}\right] \leq 0$,

$\Lambda_{\varepsilon}\left[U_{\varepsilon}-\bar{U}_{\varepsilon}\right]=0$,

$\Lambda_{\bar{u}} \geq 0 \quad \Lambda_{\varepsilon} \geq 0$.

Interpretation of this system is similar to that described for the earlier model. Up to the value of the multiplier $\Lambda_{\sigma}$, (37) expresses the constitutive properties. With $\gamma_{j}$ to represent displacement fields, (38) and (39) assure consistency in deformation kinematics (compatibility). Lastly, equilibrium conditions appear explicitly in the constraints of [PII]. In deference to space limitations, additional detail on the interpretation of the problem is not covered here. Note that [PII] also may be extended to include a load parameter, much as was done to obtain characterization [ PI.1 ]. Also, an argument similar in form to that given in the Appendix for [PI] is available to confirm that the solution to (system (37)-(43) together with the constraints of PII) is the maximizer of mixed energy $\int_{\Omega} \sigma_{i j} \varepsilon_{i j} \mathrm{~d} V$. With space limitations in mind still, the model incorporating a load parameter and the sufficiency proof for this model are not presented here. We remark that a connection between the familiar $\mathrm{Hu}$-Washizu stationarity principle (see e.g. (Washizu 1982)) and the formulation [ PII] may be observed, much as was done to interpret a relationship between the Reissner statement and $[\mathrm{PI}]$. 
2.1

\section{Applications in analysis and design}

With all the measures of elasticity directly accessible, i.e. explicit in the problem statements, the basic mixed-form formulations afford a broad range of possibilities for application. Examples described here are intended simply to be suggestive and to indicate certain techniques useful in developing applications. An extension of the simple linear elasticity model to cover stress-limited analysis and design is described. The example serves to illustrate how the addition of constraints leads to an extended portrayal of the mechanics. A treatment for optimal design problems stated in min-max form confirms that the convenience realized in certain earlier formulations for such problems is still available in connection with the mixed model. A formulation to predict the optimal stiffening of a material exemplifies another form of design optimization, in the case for which the design variable is identified with a controlled relaxation of constraints. A model for elastostatics within bounds on strain (locking material), having the form of an extension of [PII], is described as a final example.

The portrayal obtained by introduction into [PI] or [PI.1] of a constraint representing a bound on the stressstate may be interpreted to simulate overall behavior for which a degradation or softening of material occurs. For simplicity in the demonstration, the problem is described here as it would apply in 1D linear elasticity. For a bar of cross-sectional area $A$, elastic compliance $C$, and loaded over its length by $f$, the formulation is stated as

$\max _{\sigma, \varepsilon, u} \int_{0}^{L} A \sigma \varepsilon \mathrm{d} x$,

subject to

$$
\begin{aligned}
& \int_{0}^{L} f u \mathrm{~d} x-\bar{W} \leq 0, \\
& \int_{0}^{L} \frac{1}{2} A C \sigma^{2} \mathrm{~d} x-\bar{U} \leq 0, \\
& A\left(\varepsilon-u^{\prime}\right)=0, \\
& A(\sigma-\bar{\sigma}) \leq 0, \\
& A(-\sigma-\underline{\sigma}) \leq 0 .
\end{aligned}
$$

This statement is a $1 \mathrm{D}$ version of [PI] additionally constrained to reflect upper and lower bounds on stress. The representation of elastostatics is the same in the present case as was shown for [PI], except for the equation corresponding to (5), which represents stationarity w.r.t. $\sigma$. For the present stress-limited problem the latter condition is

$\varepsilon=\Lambda_{\bar{U}} C \sigma+\mu-\nu$, where $\mu$ and $\nu$ are multipliers on the respective stress constraints. These multipliers are orthogonal. According to the complementarity relations for the stress constraints, $\underline{\sigma}<\sigma<\bar{\sigma} \rightarrow \mu=\nu=0$, so where the stress lies within its limits, the above strain-stress relation is linear. On the other hand, for sigma positive and at its limit value

$\varepsilon=\Lambda_{\bar{U}} C \bar{\sigma}+\mu$.

Here $\mu$ represents the difference between the total strain and the value of the strain at the stress limit. (This corresponds to the role of an internal variable commonly introduced in plasticity theories to represent plastic strain.) $\nu$ has similar meaning for the stress on the negative side, i.e. for the stress at its lower limit

$\varepsilon=-\Lambda_{\bar{U}} C \underline{\sigma}-\nu$.

Thus the description of elasticity for a stress-limited material is complete.

Optimal design for a stress-limited material is now described simply by incorporation of the above model for the mechanics into a min-max form that covers design (see e.g. (Bendsøe 1995)). Accordingly, optimal design of $A(x)$ is expressed symbolically as

$$
\begin{aligned}
& \max _{\sigma, \varepsilon, u} \int_{0}^{L} A \sigma \varepsilon \mathrm{d} x, \\
& \min \\
& \text { A } \\
& \text { subject to } \\
& \begin{array}{c}
\underline{A}-A \leq 0, \\
\int_{0}^{L} A \mathrm{~d} x-R \leq 0,
\end{array} \\
& \text { subject to } \\
& \int_{0}^{L} f u \mathrm{~d} x-\bar{W} \leq 0, \\
& \int_{0}^{L} \frac{1}{2} A C \sigma^{2} \mathrm{~d} x-\bar{U} \leq 0, \\
& A\left(\varepsilon-u^{\prime}\right)=0, \\
& A(\sigma-\bar{\sigma}) \leq 0, \\
& A(-\sigma-\underline{\sigma}) \leq 0 .
\end{aligned}
$$

The specified $R$ represents the limit on the global measure of structural resource. The optimality condition for this problem is, after some simplification,

$\sigma \varepsilon=\frac{1}{2} \Lambda_{U} C \sigma^{2}+\Phi-\varphi$.

Symbols $\Phi$ and $\varphi$ represent multipliers associated, respectively, with the global and local constraints on the design variable $A(x)$. Upon substitution for $\varepsilon$ using the result obtained earlier, the optimality condition becomes

$\frac{1}{2} \Lambda_{U} C \sigma^{2}=\Phi-\varphi-\sigma(\mu-\nu)$.

Thus in intervals of the structure for which the stress is within its limits, the condition requires

$\frac{1}{2} \Lambda_{U} C \sigma^{2}=\Phi-\varphi$, 
i.e. the expected and familiar requirement in minimum compliance design that unit energy is bounded throughout the structure and has uniform value at the bound in intervals for which design $A$ is above its lower limit. In intervals for which upper or lower limits on stress are active, the optimality condition states, respectively,

$\frac{1}{2} \Lambda_{U} C \sigma^{2}+\bar{\sigma} \mu=\Phi-\varphi$,

and

$\frac{1}{2} \Lambda_{U} C \sigma^{2}+\underline{\sigma} \nu=\Phi-\varphi$.

The set of one or another among these relations and the above conditions holds at every point in the domain of the structure. Thus one may appreciate how they provide for all possibilities among 'stress levels within their limits', and for 'all admissible designs'. The design problem is properly posed and the often cited concern over the consequence of limitations associated with the kinematic requirements of a displacement based formulation [see e.g. the survey by Rozvany (2001)] does not arise here. We remark that, with the use of a mutual energy formulation (Taylor and Bendsøe 2001) in place of the present one, the counterpart to the results described here may be established for design w.r.t. design objectives other than compliance.

Example solutions for analysis within stress limits are furnished in (Taylor 1993) and (Plaxton and Taylor 1994); those of the latter show evolution of the stressstrain state with increasing load. Optimal design within stress limits is presented for trussed structures in (Taylor 1993) and for continuum ones in (Bendsøe et al. 1996). (The problem formulation used in these earlier works differs from the present mixed-model.) Numerical examples included in the former paper depict changes in layout with increasing load for the optimal truss, as well as the associated material distribution. The stress-limited formulation is elaborated in the next application to cover a form of design problem associated with a relaxation of the stress limits. The problem admits interpretation as a means to predict the optimal distribution of a stiffening improvement in material properties. The design problem may be compared to an earlier model for optimal remodeling (Olhoff and Taylor 1979), noting the difference that, in the present case, the modification variable is introduced in the constraints. The local measure of improvement is labeled $\rho$, and the total resource available for this purpose is represented by $R$. With the introduction of modification $\rho$ to the stress-bounds, where $\rho$ is subject to local and global constraints, the problem is stated as

$\max _{\sigma, \varepsilon, u, \rho} \int_{0}^{L} A \sigma \varepsilon \mathrm{d} x$, subject to

$$
\int_{0}^{L} f u \mathrm{~d} x-\bar{W} \leq 0 \quad \int_{0}^{L} \frac{1}{2} A C \sigma^{2} \mathrm{~d} x-\bar{U} \leq 0,
$$

$A\left(\varepsilon-u^{\prime}\right)=0$,

$A[\sigma-(\bar{\sigma}+\rho)] \leq 0$,

$A[-\sigma-(\underline{\sigma}+\rho)] \leq 0$,

$-\rho \leq 0$,

$\int_{0}^{L} \rho \mathrm{d} x-R \leq 0$.

The entire system of governing equations and inequalities of the original stress-limited problem holds in the present design example as well. The optimal distribution $\rho$ of stiffening reinforcement may be predicted as the solution of this system together with the optimality condition, i.e. the condition for stationarity w.r.t. $\rho$

$\mu+\nu+\omega=\Lambda_{\rho} \quad x \in \Omega$,

$\Omega$ represents the domain of the structure. Here $\omega$ and $\Lambda_{\rho}$ are multipliers on the last two of the constraints listed above. To interpret in part the optimality condition, note that wherever reinforcement should occur, $\rho>0 \rightarrow \omega=0$ and so $\mu+\nu=\Lambda_{\rho}, x \in \Omega_{\rho}$, where the intervals of stiffening $\Omega_{\rho}$ are identified via

$\Omega_{\rho}:=\{x \in \Omega \mid \rho>0\}$.

Recalling that $\mu$ and $\nu$ are orthogonal, the reinforcement interval is composed of segments, each associated with stiffening for which the stress is either positive or negative. Recalling the earlier interpretation of ' $\mu$ ' and ' $\nu$ ', this result indicates that, in the reinforced segments, the inelastic part of strain has uniform value. We forego covering additional detail and simply note that for the meaningful range of stiffening, $0<R<R^{*}$. At the bound value $R=R^{*}$, stress equals its limiting value at most at a finite set of points (the structure is fully reinforced). Considering application of the model to problems in 2D or $3 \mathrm{D}$, the modification variable would have the form of a reinforcement tensor. Expression of the related constraints calls for the introduction of a local invariant measure of this tensor. In this case the formulation might be elaborated to good purpose using a representation of 'cost' in generalized form (e.g. (Taylor and Washabaugh 1997)).

As was indicated at the start of this section on applications, the last example is obtained from elaboration of 
formulation [ PII ] by the addition of constraints on magnitude of strain. A material having the properties implicit in the model elaborated in this way has been termed an elastic-locking material (Prager 1964). Expressed for 1D elasticity, the problem is described by

$\max _{\sigma, \varepsilon, u} \int_{0}^{L} A \sigma \varepsilon \mathrm{d} x$,

subject to

$A u(0)=0$,

$\left.(A \sigma \bar{u})\right|_{x=L}-\bar{W} \leq 0$,

$\int_{0}^{L} \frac{1}{2} A E \varepsilon^{2} \mathrm{~d} x-\bar{U} \leq 0$,

$-\left[(A \sigma)^{\prime}+f\right]=0$,

$A(\varepsilon-\bar{\varepsilon}) \leq 0$,

$A(-\varepsilon-\underline{\varepsilon}) \leq 0$.

Here $\bar{u}, f$, and all indicated bounds are prescribed. With $\Lambda_{0}, \Lambda_{W}, \Lambda_{U}, \gamma, s$, and $t$ as multipliers, listed in order of the constraints, stationarity w.r.t. $\sigma$ and $\varepsilon$ requires

$\varepsilon=\gamma^{\prime}+s-t \quad x \in \Omega$,

$\sigma=\Lambda_{U} E \varepsilon+s-t \quad x \in \Omega$,

and the associated boundary conditions. Thus over intervals of the structure for which

$\underline{\varepsilon}<\varepsilon<\bar{\varepsilon} \quad \rightarrow \quad s=t=0$,

and with interpretation of $\gamma$ to represent displacement, the system corresponds simply to linear elasticity. On the other hand, in intervals for which either $s>0$ or $t>0$, the 'material is locked'. There the value of the stress is relaxed by amount $s$ or $t$, i.e. in these intervals, the stress differs from the value predicted for linear elasticity by these amounts. In deference to space limitations, we forego further detail on the interpretation of this problem. (The analysis with elastic-locking material is treated in greater detail in (Taylor 1994), albeit using a different formulation.) Suffice it to state that, as in the earlier examples, the problem is well posed and the governing system provides for the prediction of a unique response. Note that design problems may be considered for an elastic-locking material analogous to those treated above in the case of a stress-limited material. For example, the same technique described for relaxation of the bound values in the latter case may be used in conjunction with the elastic-locking formulation to predict optimal relaxation of the limits on strain. The resulting design describes an optimally softened structure.

\section{References}

Bendsøe, M.P. 1995: Optimization of structural topology, shape, and material. Berlin, Heidelberg, New York: Springer

Bendsøe, M.P.; Guedes, J.M.; Plaxton, S.; Taylor, J.E. 1996: Optimization of structure and material properties for solids composed of softening material. Solids Struct. 33, $1799-1813$

Hestenes, M.R. 1966: Calculus of variations and optimal control theory. New York: John Wiley \& Sons

Holzapfel, G.A. 1998: Nonlinear solid mechanics. Chichester: John Wiley \& Sons

Langhaar, H.L. 1962: Energy methods in applied mechanics. New York: John Wiley \& Sons

Mura, T.; Koya, T. 1992: Variational methods in mechanics. New York: Oxford University Press

Olhoff, N.; Taylor, J.E. 1979: On optimal structural remodeling. JOTA 27, 571-582

Plaxton, S.K.; Taylor, J.E. 1994: Application of a generalized Complementary Energy Principle for the equilibrium analysis of softening material. Comp. Methods Appl. Mech. Eng. 117, 91-103

Prager, W. 1964: On elastic, perfectly locking materials. In: Gortler, H. (ed.) Proc. 11th Int. Congr. Appl. Mech. Berlin: Springer

Reddy, J.N. 1984: Energy and variational methods in applied mechanics. New York: John Wiley \& Sons

Reissner, E. 1950: On a variational theorem in elasticity. Math. Phys. 29, 90-95

Roberts, J.E. ; J.-M. Thomas 1991: Mixed and hybrid methods In: Ciarlet, P.G; Lions, J.L. (eds.) Finite element analysis, (Part I). Amsterdam: North-Holland

Rozvany, G.I.N. 2001: Stress-ratio and compliance based methods. A critical review, Struct. Multidisc. Optim. 21, 109-119

Shames, I.H.; Dym, C.L. 1985: Energy and finite element methods in structural mechanics. New York: Hemisphere Publishing

Taylor, J.E. 1993: A global extremum principle for the analysis of solids composed of softening materials. Int. J. Solids Struct. 30, 2057-2069

Taylor, J.E. 1993: Truss topology design for elastic/softening materials. In: Bendsøe, M.P.; Mota Soares, C.A. (eds.) Topology design of structures, pp. 451-467. Dordrecht: Kluwer Academic Press

Taylor, J.E. 1994: A global extremum principle in mixed form for equilibrium analysis with elastic/stiffening material: a generalized minimum potential energy principle. J. Appl. Mech. 61, 914-918

Taylor, J.E.; Washabaugh, P.D. 1997: A generalized expression of cost for prediction of the optimal material properties 
tensor. In: Manuael Monteiro, M.D.P.; Rodrigues, J.F. (eds.) Application of mathematics to mechanics. England, Essex: Longman

Taylor, J.E.; Bendsøe, M.P. 2001: A mutual energy formulation for optimal structural design. Struct. Multidisc. Optim. 22, 95-101

Washizu, K. 1982: Variational methods in elasticity and plasticity. New York: Oxford Pergamon Press

\section{Appendix:}

As was indicated in the introduction, a proof is available to confirm that the solution to the governing conditions for either of the general mixed-form extremum principles is identified with a local maximum of the energy $\int_{\Omega} \sigma_{i j} \varepsilon_{i j} \mathrm{~d} V$. The proof for a $1 \mathrm{D}$ version of the first formulation is described in what follows; the argument for the model in 3D is the same. Thus to treat the problem [PI ], the admissible stress, strain, and displacement $\hat{\varepsilon}, \hat{\sigma}$, and $\hat{u}$ are described relative to the solution elements $\varepsilon, \sigma$, and $u$ by

$$
\begin{aligned}
& \hat{\varepsilon}=\varepsilon+e \beta, \\
& \hat{\sigma}=\sigma+e \gamma, \\
& \hat{u}=u+e \delta .
\end{aligned}
$$

Here the set of variations is restricted according to $0<e \ll 1$ and the norms of $\beta, \gamma$, and $\delta$ are bounded by unity. Accordingly, the difference in 'mixed energies' is given by

$$
\begin{aligned}
& \int_{a}^{b} A(\sigma \varepsilon-\hat{\sigma} \hat{\varepsilon}) \mathrm{d} x= \\
& -e \int_{a}^{b} A(\gamma \varepsilon+\beta \sigma) \mathrm{d} x-e^{2} \int_{a}^{b} A \beta \gamma \mathrm{d} x,
\end{aligned}
$$

Clearly the proof depends here on the term linear in $e$, i.e. it is necessary to demonstrate that the first term on the right side of the expression for the difference in energies is non-negative. To treat the first of the two parts of this term, it is assumed that $U(\hat{\sigma})$ can be expressed in terms of the expansion

$U_{0 \sigma}(\hat{\sigma})=U_{0 \sigma}(\sigma)+\left.(\hat{\sigma}-\sigma) \frac{\partial U_{0 \sigma}}{\partial \sigma}\right|_{\hat{\sigma}=\sigma}+\cdots$.
According to the second constraint of [PI],

$$
\int_{a}^{b} A U_{0 \sigma}(\sigma) \mathrm{d} x=\bar{U} ; \quad \int_{a}^{b} A U_{0 \sigma}(\hat{\sigma}) \mathrm{d} x \leq \bar{U} .
$$

Thus from the expansion and $(\hat{\sigma}-\sigma)=e \gamma$, it follows that

$-\left.e \int_{a}^{b} A \gamma \frac{\partial U_{0 \sigma}}{\partial \sigma}\right|_{\hat{\sigma}=\sigma} \mathrm{d} x \geq 0$.

Application of the necessary conditions (1) and (7), namely

$\left.\frac{\partial U_{0 \sigma}}{\partial \sigma}\right|_{\hat{\sigma}=\sigma}=\varepsilon / \Lambda_{f} \& \Lambda_{f}>0$,

leads to the conclusion

$-e \int_{a}^{b} A \gamma \varepsilon \mathrm{d} x \geq 0$.

To treat the second part of the term linear in $e$, i.e. $-e \int_{a}^{b} A \sigma \beta \mathrm{d} x$, note from the necessary conditions (2) and (3) that $A \sigma^{\prime}=-\Lambda_{f} f$. The integral over the structure of the product of displacement $u$ and this (equilibrium) equation, together with (8), leads to

$\int_{a}^{b}(A \sigma)^{\prime} u \mathrm{~d} x=-\Lambda_{f} \int_{a}^{b} f u \mathrm{~d} x=-\Lambda_{f} \bar{W}$.

By similar means but with $u$ replaced by $\hat{u}$, we find that

$\int_{a}^{b}(A \sigma)^{\prime} \hat{u} \mathrm{~d} x=-\Lambda_{f} \int_{a}^{b} f \hat{u} \mathrm{~d} x \geq-\Lambda_{f} \bar{W}$,

whereby

$\int_{a}^{b}(A \sigma)^{\prime}(u-\hat{u}) \mathrm{d} x \leq 0$.

Integration leads to

$\left.A \sigma(u-\hat{u})\right|_{B \mathrm{~d} y}-\int_{a}^{b} A \sigma(\varepsilon-\hat{\varepsilon}) \mathrm{d} x \leq 0$.

The boundary term has value zero, and substitution for the difference in strains results in

$-e \int_{a}^{b} A \sigma \beta \mathrm{d} x \geq 0$,

to complete the proof. 